\title{
GAMMA-RAY BURSTERS AS SOURCES OF COSMIC RAYS
}

\author{
Mordehai Milgrom and Vladimir Usov \\ Department of condensed-matter physics, Weizmann Institute, Rehovot 76100 Israel
}

The little we do know of the physical conditions in $\gamma$-ray bursters 1 makes them conducive to the acceleration of high-energy cosmic rays (CRs), especially if they are at cosmological distances. We find that, with the observed statistics and fluxes of $\gamma$-ray bursts, cosmological bursters may be an important source of cosmic rays in two regions of the observed spectrum: 1. At the very-high-energy end $\left(E>10^{19} \mathrm{eV}\right)$, where CRs must be of extragalactic origin. 2. Around and above the spectral feature that has been described as a bump and/or a knee, which occurs around $10^{15} \mathrm{eV}$, and starts at $E_{0} \sim(1-3) \times 10^{14} \mathrm{eV}$. The occasional bursters that occur inside the Galaxy-about once in a few hundred thousand years if burst emission is isotropic; more often, if it is beamed-could maintain the density of galactic cosmic rays at the observed level in this range. These two energy ranges might correspond to two typical CR energy scales expected from bursters: one pertinent to CR acceleration due to interaction of a magnetized-fireball front with an ambient medium; the other to acceleration in the fireball itself (e.g. shock acceleration).

Cosmic rays are thought to originate from more than one type of source, but what exactly their sources are is still an unsettled issue (for recent reviews see e.g., refs [2] - [4]). Those with energies above $\sim 10^{19} \mathrm{eV}$ must be extragalactic, because their gyroradius in galactic magnetic fields is larger than galactic size. Those of lower-energy, which are of galactic origin, also seem to come from more than one type of source 4 , as indicated by the following observations: 1 . The change in the spectral form above $E_{0}$ may witness a change in type of source, or at least in the acceleration mechanism. 2. Supernova remnants (SNRs), which, on various grounds, are thought to be adequate sources for the lower energy CRs, seem incapable 5 of producing the higher-energy galactic CRs with $E>10^{14} \mathrm{eV}$. It may be significant that this theoretical cutoff at $\sim 10^{14} \mathrm{eV}$ marks the beginning of the observed change in both the spectrum, and the elemental composition. After Axford we designate the former, sub- $E_{0}$ region GCRI, and the latter by GCRII. It is widely acknowledged (e.g. refs [3, 4]) that the origins of both GCRII and extragalactic CRs (EGCRs) have 
not been pinpointed yet. Below, we argue that $\gamma$-ray-burst sources (GRBSs) may be suitable sources of both these components of CRs.

Milgrom and Usov 6 have recently pointed out that the directions of arrival of the two most energetic CRs observed to date each agree, within the uncertainties, with that of an energetic $\gamma$-ray burst (GRB). Inspired by this we have proposed that ultra-high-energy CRs $\left(E>10^{20} \mathrm{eV}\right)$ are accelerated in the same cataclysmic event that produces the GRB (the CRs arriving with some delay, and positional disparity due to wriggling of its trajectory by intervening magnetic fields). Waxman [7, and Vietri 8 have made a similar proposal. This has, naturally, induced us to consider the contribution of GRBSs to the observed CR population at lower energies.

The isotropy in the directions of arrival of $\gamma$-ray bursts, together with the distance distribution implied by their $\log N-\log S$ relation, points strongly to a GRB origin far outside the disk of the Milky Way. This has lead to two alternative pictures: one in which GRBs are of cosmological origin - 12 and one by which their sources are distributed in a spherical, extended corona, surrounding the Milky Way 13 - [15]. We adopt the cosmological scenario in the following discussion; the galactic-corona alternative will be discussed, occasionally.

One has to consider two types of cosmic-ray contribution: 1. CRs at very high energies that are hardly affected by magnetic fields in their putative mother galaxy, and in our own, arrive here as extragalactic CRs. 2. The occasional GRBSs that occur in the Milky Way (still in the cosmological scenario for GRBs) produce a flux of high-energy CRs passing Earth on their way out to become extragalactic CRs in other galaxies; these we ignore here. They also emit CRs of low enough energies to be trapped in the Galaxy for an extended period and contribute to its CR budget. We first consider such trapped CRs from bursts inside the Galaxy.

The observed energy density of all CRs with $E>10^{10} \mathrm{eV}$ in the neighborhood of the solar system is $w_{\mathrm{CR}} \simeq 0.5 \mathrm{eV} \mathrm{cm} \mathrm{cm}^{-3}$. For maintaining this energy density, in spite of CR escape, a refilling of the galactic volume by CRs with a power $Q_{\mathrm{CR}}^{I} \simeq$ $3 \times 10^{40} \mathrm{erg} \mathrm{sec}^{-1}$ is required (see e.g. [16]). As to GCRII, the power which is needed to maintain the observed energy density, $w_{\mathrm{CR}}^{I I}$ of CRs with energy above $E_{0}$ is estimated at

$$
Q_{\mathrm{CR}}^{I I} \simeq \frac{V_{\mathrm{CR}} w_{\mathrm{CR}}^{I I}}{\tau_{\mathrm{conf}}\left(E_{0}\right)} \simeq Q_{\mathrm{CR}}^{I}\left(\frac{E_{0}}{10^{10} \mathrm{eV}}\right)^{-(\gamma-2)+\eta} \quad \mathrm{erg} \mathrm{sec}^{-1} .
$$

Here $V_{\mathrm{CR}}=\pi R_{\mathrm{g}}^{2} h_{\mathrm{CR}} \simeq 10^{67}\left(h_{\mathrm{CR}} / h_{\mathrm{rad}}\right) \mathrm{cm}^{3}$ is the volume where CRs accumulate; $R_{\mathrm{g}} \simeq 10 \mathrm{kpc}$ is some measure of the radius of the Galaxy, $h_{\mathrm{CR}}$ is the thickness of the $\mathrm{CR}$ volume; $h_{\mathrm{rad}} \simeq 750 \mathrm{pc}$ is the thickness of the radio disk; $\gamma \simeq 2.7$ is the spectral power of CRs from $10^{10} \mathrm{eV}$ to $\sim E_{0}$ (ref. 四); so that $w_{\mathrm{CR}}^{I I} \simeq w_{\mathrm{CR}}\left(E_{0} / 10^{10} \mathrm{eV}\right)^{-(\gamma-2)}$. We have taken an energy dependence of the confinement time of the form 


$$
\tau_{\text {conf }}(E) \simeq 10^{7}\left(\frac{h_{\mathrm{CR}}}{h_{\mathrm{rad}}}\right)\left(\frac{E}{10^{10} \mathrm{eV}}\right)^{-\eta} \mathrm{yr}
$$

and $\eta=0.4 \pm 0.2$ (refs $[17]$ - [19 ). Actually, very little is known about the confinement time for energies outside the range $10^{10}-10^{12} \mathrm{eV}$, where it can be measured approximately from the abundance of radioactive secondaries among the CRs. In this range one result is $17 \simeq 0.6$. More generally, it was argued 18 19 that in a wide range of energies, from $\sim 10^{9} \mathrm{eV}$ to $\sim 10^{17} \mathrm{eV}$, the mean value of $\eta$ is about $0.3 \pm 0.1$. If we use $\eta=0.3$ and $E_{0}=3 \times 10^{14} \mathrm{eV}$ we have from eq.(1)

$$
Q_{\mathrm{CR}}^{I I} \simeq 5 \times 10^{38} \mathrm{erg} \mathrm{sec}^{-1} \text {. }
$$

While the value of $h_{\mathrm{CR}}$ is very uncertain, and can be anywhere between $\sim h_{\text {rad }}$ and $\sim 2 R_{\mathrm{g}}$ (e.g. ref. [16]), our estimate of $Q_{\mathrm{CR}}^{I I}$ does not depend on it.

What fraction of such a CR luminosity is reasonable of GRBSs to supply? To get an idea we estimate the long-time-mean luminosity in $\gamma$-rays produced by GRBSs inside the Galaxy. From the typical distances (as inferred from observed statistics), and from the fluxes of GRBs, it is estimated (e.g. refs [1] 20] [21]) that, if the $\gamma$-rays are emitted isotropically from their sources, an average GRBS outputs $\sim 4 \times 10^{51}$ ergs in $\gamma$-rays; and that if GRBSs occur in galaxies with rates proportional to the galactic mass, about one GRB occurs in a galaxy like ours once every $\sim 4 \times 10^{5}$ years. If the $\gamma$-ray emission is beamed the luminosities are smaller, in proportion, and the rate of occurrence in a galaxy is higher, in inverse proportion, to the beam solid angle, which might be rather small. By these estimates the mean power that is released in CRs in the Galaxy from bursts inside it is $L_{\mathrm{CR}} \simeq 3 \times 10^{38} \alpha \mathrm{erg} \mathrm{sec}^{-1}$, where $\alpha$ is some typical ratio of CR-to- $\gamma$-ray total energy output. Thus if $\alpha$ is of order unity the CR luminosity produced by the GRBs in the Galaxy may tally with what is needed to maintain the observed $C R$ flux in GCRII by eq.(3). In the galactic-corona-origin picture similar arguments require $\alpha>10^{4}$.

To achieve the observed degree of isotropy of GCRII is not much more difficult than with other type of sources, say SNRs; even though GRBSs inject CRs in short, far-between bursts in space and time. Especially so as that the confinement time may be much larger than the repetition time of the GRBSs in the Galaxy.

Consider now the possible contribution of GRBSs to the observed EGCR. The reckoning here is different: we now compare the integrated flux of $\gamma$-ray bursts with that of CRs of energy $E>10^{19} \mathrm{eV}$. We assume that intergalactic magnetic fields are small enough so that such CRs are not accumulated in the IGM. The integral energy flux of EGCRs is $I_{\mathrm{CR}}\left(E>10^{19} \mathrm{eV}\right) \simeq 4 \times 10^{-10} \mathrm{erg} \mathrm{cm}^{-2} \mathrm{~s}^{-1}$ above $10^{19} \mathrm{eV}$ and $I_{\mathrm{CR}}\left(E>10^{20} \mathrm{eV}\right) \simeq 0.6 \times 10^{-10} \mathrm{erg} \mathrm{cm}^{-2} \mathrm{~s}^{-1}$ above $10^{20} \mathrm{eV}$ (ref [22]). The energy flux in GRBs is $I_{\gamma}=\dot{N} S_{0} \simeq 3 \times 10^{-10} \mathrm{erg} \mathrm{cm}^{-2} \mathrm{~s}^{-1}$, where $\dot{N} \simeq 10^{3} \mathrm{yr}^{-1}$ is the total observed burst rate and $S_{0} \simeq 10^{-5} \mathrm{erg} \mathrm{cm}^{-2}$ is a typical fluence per burst, as derived from the BATSE survey (e.g., ref [1]). From these estimates we can see that the 
flux of EGCRs with $E \sim 10^{19} \mathrm{eV}$ may be produced by GRBSs if their luminosities in $\gamma$-rays and CRs of energies $\sim 10^{19} \mathrm{eV}$ are similar: $\alpha \sim 1$ at $E \sim 10^{19} \mathrm{eV}$. The fact that the estimated, integrated flux of CRs above $10^{20} \mathrm{eV}$ is rather smaller than that above $10^{19} \mathrm{eV}$ does not indicate that the spectrum is so steep at the source, as the higher-energy CRs suffer effective degradation of energy on their way. In fact, observations are consistent with an $E^{-2}$ energy spectrum that imparts equal energies to equal logarithmic ranges. (See also the discussion by Waxman [7] who reaches similar conclusions on this point).

The observed isotropy in the directions to GRBSs 1 , would naturally explain the near isotropy of EGCRs, if these indeed are produced by GRBSs. There may be an added isotropization effect due to Larmor bending of the trajectories of the lowerenergy EGCRs by intergalactic magnetic fields. (The limits on such fields are so lax as to be consistent with either an important such effect, or with hardly any.) However, CRs with the highest energies, say $E>5 \times 10^{19} \mathrm{eV}$, cannot, typically, come from very large distances, because their energy is quickly degraded on travel 23 . Their positional distribution will than reflect that of the nearer-by GRBSs-at these energies the CR apparent position has a good memory of the direction of the source (e.g. ref. 223). If GRBSs occur in galaxies, the CR distribution will reflect any anisotropy in the distribution of nearby galaxies (distance less than 200-300 Mpc). Indeed, such an anisotropy for $E>4 \times 10^{19} \mathrm{eV}$, correlated with the local supercluster has been found recently 24. GRBSs within this distance are also far-between in time (a few per year). Thus we could see $E>10^{20} \mathrm{eV}$ CRs to come in groups spread in time over periods of a few months to a few years (see ref. [6]), and concentrated in an area of the sky around the GRBS position. This could be tested in future with the proposed $5000 \mathrm{~km}^{2} \mathrm{CR}$ detector, the Auger observatory, with which a few tens such CRs are expected for these strongest GRBS.

What are the typical CR energies expected from GRBSs, and what are the relative efficiencies, $\alpha$, of CR to $\gamma$-ray productions, which enter our deductions of the expected CR fluxes from $\gamma$-ray observations?

There are two energy scales that might characterize the energy of cosmic rays accelerated in GRBSs; one is $\Gamma_{0}^{2} m c^{2}$, where $\Gamma_{0}$ is the bulk Lorentz factor of a relativistic wind, and $m$ the mass of the particle; the other may be defined, for example by the electric potential produced by rotating magnetic fields so that it depends on the magnetic-field strength, and the rotation frequency. The first scale enters as follows: A common feature of all acceptable models of cosmological GRBSs is that a relativistic wind is involved in the $\gamma$-ray radiation process. The bulk Lorentz factor of such a wind is constrained to be more than $10^{2}-10^{3}$ (refs [21], 25]). A very strong magnetic field may exist in the plasma that flows away from GRBSs 26 27. It was argued by Mészáros and Rees 28 that the X-ray and $\gamma$-ray emission of bursts is generated far from the bursters, at distances of $r_{\mathrm{d}} \sim 10^{15}-10^{16} \mathrm{~cm}$, in the process of interaction between relativistic winds with $\Gamma_{0} \geq 10^{2}$ and an external medium (e.g., an ordinary interstellar medium, or plasma that is ejected from the 
predecessor of the burster). Such a process has been considered numerically 29], and it was shown that the energy that is lost by a relativistic magnetized wind because of its interaction with an external medium is distributed in the following way. About half of this energy is in ultra-relativistic heavy particles, protons and nuclei that are knocked by the wind front. In the rest frame of the front the particles are reflected, more-or-less elastically, from the front. Thus, the mean Lorentz factor of reflected heavy particles in the frame of the burster is $\sim \Gamma_{0}^{2}$. The other half of the wind-energy losses is distributed, more-or-less evenly, between low-frequency oscillations of electromagnetic fields and both high-energy electrons and their high-frequency (X-ray and $\gamma$-ray) emission. The total $\gamma$-ray efficiency may reach $20-30 \%$. Therefore, it is estimated that the energy released in CRs is at least twice that in $\gamma$-rays: $\alpha \geq 2$. For this lower limit the estimated mean power released in CRs in the Galaxy from local GRBSs is $L_{\mathrm{CR}} \simeq 6 \times 10^{38} \mathrm{erg} \mathrm{sec}^{-1}$, which is comparable to $Q_{\mathrm{CR}}^{I I}$ within uncertainties of their estimates.

The mean energy of CRs knocked by the magnetized-fireball front would be the typical energy of the bump, $\sim 10^{15} \mathrm{eV}$ per nucleus, if $\Gamma_{0} \sim 10^{3}$, which is much in keeping with what is expected; but, $\Gamma_{0}$ may be as high as $10^{5}$ (ref. 28]) giving CR energies of up to $\sim 10^{19} \mathrm{eV}$ per nucleus. Hence, the occasional GRBSs that occur in the Milky Way might explain both the energetics, and the typical energy of GCRII. Our limited knowledge of the processes involved does not suffice do determine the expected spectrum of CRs.

The second energy scale enters because CRs may be generated by mechanisms other than through knocking by the relativistic interface between the outflowing gas and an external medium, as described above. For example, by one class of cosmological-origin models, GRBs are produced in rotating disk-like objects resulting from mergers of a neutron-star binaries (e.g. ref. [30]), or by neutron stars formed by accretion-induced collapse 27. The magnetic field, $B_{\mathrm{s}}$, at the surface of such objects may be as high as $\sim 10^{16} \mathrm{G}$ (refs [26], [27]), and their angular velocity $\Omega \sim 10^{4} \mathrm{~s}^{-1}$. The potential difference between the surface of such an object, at radius $R \sim 10^{6} \mathrm{~cm}$, and infinity is 31

$$
\Delta \varphi_{\max }=\frac{\Omega^{2} B_{\mathrm{S}} R^{3}}{2 c^{2}} \sim 10^{23}\left(\frac{\Omega}{10^{4} \mathrm{~s}^{-1}}\right)^{2}\left(\frac{B_{\mathrm{S}}}{10^{16} \mathrm{G}}\right)\left(\frac{R}{10^{6} \mathrm{~cm}}\right)^{3} \mathrm{~V} .
$$

Charged particles that flow away from the surface may be accelerated, in principle, up to the energy $E_{\max } \simeq e \Delta \varphi_{\max }$, enough to explain EGCRs of all observed energies. Alternatively, particles may be accelerated by relativistic shocks that may be formed in an unsteady relativistic wind [32 up to similar energies (see e.g. refs [6, 7], \&]).

For very-high-energy CRs (termed EGCR) to originate in coronal GRBSs requires a CR to $\gamma$-ray ratio $\alpha \sim 1$, but existing physical GRBS models (e.g. ref. [15]) are incapable of producing CRs with such high energies. 


\section{References}

[1] Hartmann, D. in The Gamma-Ray Sky with Compton GRO and SIGMA, eds. M.Signore, P. Salati, \& G. Vedrenne (in the press).

[2] Biermann, P.L. in 23rd Intl. Cosm. Ray Conf., Calgary, Inv., Rap., High. Papers, 45-51 (1993).

[3] Drury, L.O'C. Contemporary Phys. 35, 231-242 (1994).

[4] Axford, W.I. Astrophys. J. Suppl. Ser. 90, 937-944 (1994).

[5] Blandford, R.D. \& Eichler, D. Phys. Reports 154, 1-75 (1987).

[6] Milgrom.M \& Usov, V.V. Astrophys. J. Lett. (in the press).

[7] Waxman, E. Phys. Rev. Lett. (in the press).

[8] Vietri, M. Astrophys. J. (in the press).

[9] Usov, V.V. \& Chibisov, G.V. Soviet Astr. 19, 115-116 (1975).

[10] Paczyński, B. Asta Astr. 41, 257-267 (1991).

[11] Piran, T. Astrophys. J. 389, L45-L48 (1992).

[12] Tamblyn, P. \& Melia, F. Astrophys. J. 417, L21-L24 (1993).

[13] Fishman, G.J. et al. Astrophys. J. 223, L13-L15 (1978).

[14] Li, H. \& Dermer, C.D. Nature 359, 514-516 (1992).

[15] Podsiadlowski, P., Rees, M.J. \& Ruderman, M. Mon. Not. R. astr. Soc. 273, 755-771 (1995).

[16] Berezinskii, V.S., Bulanov, S.V., Dogiel, V.A., Ginzburg, V.L. \& Ptuskin, V.S. Astrophysics of Cosmic Rays (North-Holland, Amsterdam, 1990).

[17] Müller, D. et al. Astrophys. J. 374, 356-365 (1991)

[18] Biermann, P.L. Astr. \& Astrophys. 271, 649-661 (1993)

[19] Ptuskin, V.S. et al. Astr. \&5 Astrophys. 268, 726-735 (1993)

[20] Cohen, E. \& Piran, T. Astrophys. J. Lett. 444, L25-L28 (1995).

[21] Woods, E. \& Loeb, A. Astrophys. J. (in the press). 
[22] Brooke, G., et al. Proc. 19th Intl. Cosmic Ray Conf. (La Jolla) 2, 150-153 (1985).

[23] Sigl, G., Schramm, D.N., \& Bhattacharjee, P. Astropart. Phys., 2, 401-411 (1994).

[24] Stanev, T., Biermann, P.L., Lloyd-Evans, J., Rachen, J.P., \& Watson, A. Phys. Rev. Lett. (submitted).

[25] Baring, M.G. Astrophys. J. Suppl. Ser. 90, 899-903 (1993).

[26] Narayan. R., Paczyński, B. \& Piran. T. Astrophys. J. 395, L83-L86 (1992).

[27] Usov, V.V. Nature 357, 472-474 (1992).

[28] Mészáros, P. \& Rees, M.J. Astrophys. J. 405, 278-284 (1993).

[29] Smolsky, M.V. \& Usov, V.V. Astrophys. J. (submitted).

[30] Piran, T. in Gamma-Ray Bursts, Second Workshop, (eds. G.J. Fishman, J.J. Brainerd, \& K. Hurley) 543-546 (AIP, New York, 1994).

[31] Ruderman, M.A. \& Sutherland, P.G. Astrophys. J. 196, 51-72 (1975).

[32] Rees, M.J., \& Mészáros, P. Astrophys. J. 430, L93-L96 (1994). 